\title{
CONCENTRATION-DEPENDENT DIFFUSION III. AN APPROXIMATE SOLUTION*
}

\author{
By L. F. SHAMPINE (Sandia Laboratories, Albuquerque)
}

In the papers $[1,2]$, hereafter referred to as I and II respectively, we discussed the similarity solution of the one-dimensional diffusion equation $c_{t}=\left(D c_{x}\right)_{x}$ when the diffusion coefficient $D$ is a function of the concentration $c$. The physical problem that concerns us is the diffusion of a substance into a semi-infinite medium after the substance is introduced at the face at a given concentration level which is maintained thereafter. Normalizing and introducing the similarity variable $\eta=x / \sqrt{ } t$ leads to the problem

$$
\begin{gathered}
\frac{d}{d \eta}\left(D(c) \frac{d c}{d \eta}\right)+\frac{\eta}{2} \frac{d c}{d \eta}=0, \\
c(0)=1, \quad c(\infty)=0 .
\end{gathered}
$$

In addition to $(1,2)$ a solution must have a continuous flux $f(\eta)=D(c(\eta)) c^{\prime}(\eta)$. We suppose $D(c) \in C^{1}[0,1]$ and that $D(c)>0$ for $c>0$. If $D(0)=0$, the problem is said to be singular and there may then be an $\eta_{0}$ such that the solution $c(\eta) \equiv 0$ for $\eta \geq \eta_{0}$. This $\eta_{0}$ represents the location of a "front" of a nonlinear wave of the diffusant.

Bounds on the initial flux of the solution are useful in two ways. They were necessary in I and II for computing the solution. They are quite useful physically because of their relation to the uptake, which is a quantity readily measured: the total amount of diffusant which has crossed $x=0$ by time $t, M_{t}$, is

$$
M_{\imath}=\int_{0}^{\infty} c(x, t) d x=\sqrt{ } t \int_{0}^{\infty} c(\eta) d \eta
$$

Using the conservation law

$$
-f(0)=\frac{1}{2} \int_{0}^{\infty} c(\eta) d \eta
$$

which we proved for both singular and non-singular cases, we see that

$$
M_{t}=-f(0) 2 \sqrt{ } t .
$$

By physical reasoning Macey [3] derived an approximate solution of $(1,2)$ which in our notation is

$$
\begin{gathered}
f^{*}=-\left(\frac{1}{2} \int_{0}^{1} \tau D(\tau) d \tau\right)^{1 / 2} \\
\eta^{*}(c)=\left(\int_{c}^{1} D(\tau) d \tau\right) /\left(-f^{*}\right) \text { for } c \geq 0 .
\end{gathered}
$$

* Received August 23, 1974. 
Here $f^{*} \doteq f(0)$. He observes that in his computations $f^{*}>f(0)$, and we reported the same observation in II. This is plausible from the physical arguments. The approximation $c^{*}(\eta)$ is defined implicitly by (5) and is supplemented by $c^{*}(\eta) \equiv 0$ for $\eta \geq \eta_{0}{ }^{*}$ where

$$
\eta_{0}^{*}=\left(\int_{0}^{1} D(\tau) d \tau\right) /\left(-f^{*}\right) .
$$

In this note we shall prove by simple means that the quantity $f^{*}$ of (4) is an upper bound on the initial flux. Furthermore, if any $f^{*}$ which is a lower bound for the initial flux is used in (5), the approximation $c^{*}(\eta)$ so defined satisfies $c(\eta)>c^{*}(\eta)$ for all $\eta>0$. Lower bounds are easy to obtain, e.g. in II we derived $f(0) \geq-(\Delta / \pi)^{1 / 2}$ where $\Delta=\max _{0 \leq c \leq 1} D(c)$. Study of Macey's plots of the approximation $c^{*}(\eta)$ of $(4,5)$ shows that in every case there is a number $\delta$ such that for $0<\eta<\delta, c^{*}(\eta)>c(\eta)$ and for $\delta<\eta, c^{*}(\eta)<c(\eta)$. We shall prove this qualitative behavior is always true. In particular, we shall prove that if the problem exhibits a front at $\eta_{0}$, then $\eta_{0}$ is at least as large as the $\eta_{0}{ }^{*}$ of $(6)$.

In I and II we proved there is a unique solution to $(1,2)$ which strictly decreases from 1 to 0 . Introducing the flux as a dependent variable and the concentration as the independent variable, we see that the solution satisfies, for $1 \geq c>0$,

$$
\begin{gathered}
d \eta / d c=D(c) / f, \quad \eta(1)=0, \\
d f / d c=-\eta / 2, \quad 0>f(1) \text { given. }
\end{gathered}
$$

Now the approximation (5) is the solution of

$$
\begin{gathered}
d \eta^{*} / d c=D(c) / f^{*}, \quad \eta^{*}(1)=0, \\
d f^{*} / d c=0, \quad 0>f^{*}(1)=f^{*} \text { given }
\end{gathered}
$$

(Since $f^{*}(c) \equiv f^{*}$, we shall just write $f^{*}$ in what follows.) The flux $f(c)$ obviously is strictly increasing as $c$ decreases to 0 and in I and II it is shown to tend to zero. Evidently if $0>f(1) \geq f^{*}$, we shall have $f(c)>f^{*}$ for all $c<1$. But then

$$
d\left(\eta-\eta^{*}\right) / d c=D(c)\left(1 / f-1 / f^{*}\right)
$$

is negative for $c<1$ which implies $\eta(c)>\eta^{*}(c)$. Returning to $c$ as the dependent variable, we have shown that if $f^{*}$ is a lower bound for the initial flux, then the approximation $c^{*}(\eta)$ of $(5)$ is a strict lower bound for $c(\eta)$ as long as $c(\eta)$ is positive.

The way Macey chooses the initial flux $f^{*}$ is to insist $c^{*}(\eta)$ satisfy

$$
-f^{*}=\frac{1}{2} \int_{0}^{\infty} c^{*}(\eta) d \eta
$$

so as to imitate the behavior (3) of the true solution. This requirement results in the expression (4). It is now easy to see that $f^{*}$ so chosen is an upper bound for the initial flux. If it were not, the preceding analysis would imply $c(\eta)>c^{*}(\eta)$ as long as $c(\eta)>0$, hence

$$
-f^{*}=\frac{1}{2} \int_{0}^{\infty} c^{*}(\eta) d \eta<\frac{1}{2} \int_{0}^{\infty} c(\eta) d \eta=-f(0),
$$

and $0>f^{*}>f(0)$, contrary to our assumption.

If we suppose that $f^{*}>f(1)$, then since $f(c)$ strictly increases to zero as $c$ decreases, 
there is a unique point $\zeta$ for which $f(\zeta)=f^{*}$. Thus, according to $(7), \eta^{*}(c)-\eta(c)$ strictly increases as $c$ decreases from 1 to $\zeta$ and then strictly decreases as $c$ decreases from $\zeta$ to 0 . Returning to the concentration as the dependent variable, we have that $c^{*}(\eta)$ cannot intersect $c(\eta)$ more than once. However, using Macey's approximation $c^{*}(\eta)$ must cross $c(\eta)$ at least once. Otherwise we would have $c^{*}(\eta)>c(\eta)$ for all $\eta>0$ which implies

$$
-f^{*}=\frac{1}{2} \int_{0}^{\infty} c^{*}(\eta) d \eta>\frac{1}{2} \int_{0}^{\infty} c(\eta) d \eta=-f(0),
$$

and the consequence that $f(0)>f^{*}$ is a contradiction. Thus there is a $\delta>0$ such that $c^{*}(\eta)>c(\eta)$ for $0<\eta<\delta$ and $c^{*}(\eta)<c(\eta)$ for $\delta<\eta$.

We have seen that any $f^{*}$ less than the initial flux leads to a $c^{*}(\eta)$ from (5) which is less than $c(\eta)$. Naturally, then, the $\eta_{0}{ }^{*}$ of $(6)$ is a lower bound for the location $\eta_{0}$ of a front of $c(\eta)$, should one exist. The qualitative picture of Macey's approximation shows that using the $f^{*}$ of (4) leads to an $\eta_{0}{ }^{*}$ of (6) which must also be a lower bound for $\eta_{0}$. The $\eta_{0}{ }^{*}$ of $(6)$ is an increasing function of $f^{*}$ so Macey's approximation gives the best bound.

\section{REFERENCES}

[1] L. F. Shampine, Concentration-dependent diffusion, Quart. Appl. Math. 30, 441-452 (1973)

[2] — Concentration-dependent diffusion II. Singular problems, Quart. Appl. Math. 31, 287-293 (1973)

[3] R. I. Macey, A quasi-steady state approximation method for diffusion problems: I. Concentrationdependent diffusion coefficients, Bull. Math. Biophys. 21, 19-32 (1959) 